hep-ph/9712414

SINP/TNP/97-23

\title{
Optical activity of a neutrino gas
}

\author{
Subhendra Mohanty \\ Physical Research Laboratory \\ Navrangpura, Ahmedabad 380009, INDIA \\ José F. Nieves \\ Laboratory of Theoretical Physics \\ Department of Physics, University of Puerto Rico \\ P.O. Box 23343, Rio Piedras, Puerto Rico 00931-3343 \\ Palash B. Pal \\ Theory Group, Saha Institute of Nuclear Physics \\ Block-AF, Bidhan-Nagar, Calcutta 700064, INDIA
}

December 1997

\begin{abstract}
For photons that propagate through a gas of neutrinos with a non-zero chemical potential, the left-handed and right-handed polarization modes acquire different dispersion relations. This is due to the $C P$ and $C P T$ odd terms induced by such a background on the photon self-energy. We present a detailed calculation of this effect, which does not depend on any physical assumptions beyond those of the standard electroweak model. Some possible cosmological and astrophysical implications of our results are considered in several contexts, including the recent discussions regarding the rotation of the plane of polarization of electromagnetic waves over cosmological distances.
\end{abstract}

\section{Introduction}

It was shown some time ago by two of the present authors that, in an isotropic medium, the most general parameterization of the photon polarization tensor $\Pi_{\mu \nu}$ admits three invariant form factors. This is due to the fact that, in addition to the the photon momentum $k^{\mu}, \Pi_{\mu \nu}$ depends also on the velocity four vector 
of the center of mass of the background medium, which we denote by $u^{\mu}$. Thus, the most general form of $\Pi_{\mu \nu}$ consistent with the gauge invariance conditions

$$
k^{\mu} \Pi_{\mu \nu}(k)=0, \quad k^{\nu} \Pi_{\mu \nu}(k)=0
$$

is

$$
\Pi_{\mu \nu}(k)=\Pi_{T} R_{\mu \nu}+\Pi_{L} Q_{\mu \nu}+\Pi_{P} P_{\mu \nu}
$$

where

$$
\begin{aligned}
R_{\mu \nu} & =g_{\mu \nu}-\frac{k_{\mu} k_{\nu}}{k^{2}}-Q_{\mu \nu} \\
Q_{\mu \nu} & =\frac{\widetilde{u}_{\mu} \widetilde{u}_{\nu}}{\widetilde{u}^{2}} \\
P_{\mu \nu} & =\frac{i}{K} \varepsilon_{\mu \nu \lambda \rho} k^{\lambda} u^{\rho}
\end{aligned}
$$

with

$$
K \equiv \sqrt{\omega^{2}-k^{2}}, \quad \omega \equiv k \cdot u
$$

In Eq. (1.2), $\Pi_{T, L, P}$ are scalar functions of the Lorentz invariants $K$ and $\omega$, which have the interpretation of being the momentum and energy of the photon in the frame in which the medium is at rest. The functions $\Pi_{T, L}$ are related to the well-known dielectric and magnetic permeability functions of the medium [1], while $\Pi_{P}$ is related to a third constant that is responsible for natural optical activity. This third constant, which was called the activity constant in Ref. [2], can arise only as a result of parity $(P), C P$ as well as $C P T$ asymmetric effects. This does not necessarily mean that these symmetries have to be violated at a fundamental level. The desired asymmetries may also come from the background medium. In particular, the $P$ and $C P$ breaking effects may arise either from the violation of these symmetries at the level of the fundamental interactions, or from $P$ and $C P$ asymmetries in the background. The $C P T$ breaking effect on the other hand, must necessarily arise from the $C P T$ asymmetries in the background. Thus, for example, since the weak interactions violate $P$ and $C P$ at some level, and since normal matter is $C P T$-asymmetric, the activity constant is present in all normal matter.

The activity constant must also have a non-zero value in an asymmetric background of neutrinos and antineutrinos. In this case, parity is broken by the neutrino interactions. In addition, the required $C P$ and $C P T$ asymmetries arise if the neutrino gas has a chemical potential, so that the number of neutrinos and antineutrinos in the background is not the same. For such a system, $\Pi_{P}$ is then expected to depend on $n_{\nu}-n_{\bar{\nu}}$.

This effect can have in principle a number of interesting physical implications. For example, the standard big bang model predicts a primordial neutrino 


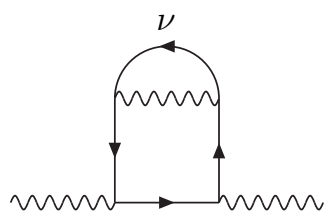

(a)

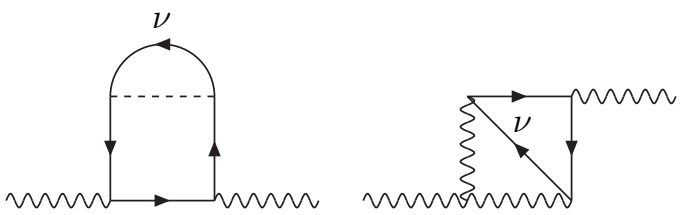

(b)

(c)

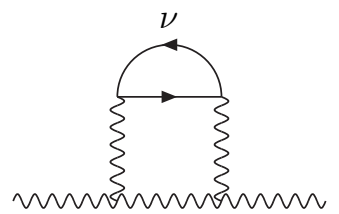

(e)

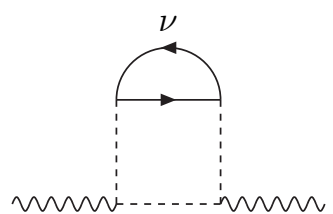

(f)

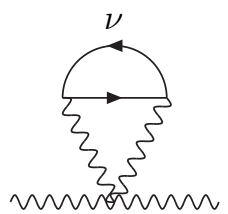

(g)

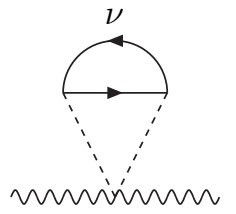

(h)

Figure 1: Two-loop diagrams for the photon self-energy, with neutrinos appearing as internal lines. The neutrino lines are marked as such. All other internal fermion lines correspond to the charged lepton that couples to the neutrino. The external gauge boson lines correspond to photons. All internal gauge boson lines are $W$-lines, and the internal scalar lines denote the unphysical Higgs boson corresponding to the $W$-boson. In addition, there are crossed diagrams obtained by interchanging the external photon lines.

background with a density comparable to that of the microwave background radiation. Light from all astrophysical sources travel through this background before reaching us and, if the background induces some optical activity, it could have interesting physical effects on this light.

In this paper we present the calculation of the activity constant for a background of neutrinos and antineutrinos. We do not assume any additional properties of the neutrinos beyond those of the standard electroweak model so that, in particular, the neutrinos are assumed to be massless, two-component objects. In contrast to the situations in which some physical effects depend on unknown or conjectured properties of the neutrinos, the implications that can be deduced from the results of our calculations are based on the well tested grounds of the standard model. While the possible observational effects may be small in particular physical situations, the effects are generally present at some level and can manifest themselves in unexpected circumstances. 


\section{Strategies for the calculation}

The basic technique for determining $\Pi_{P}$, which has already been described in Ref. [2], involves the calculation of the photon self-energy diagrams involving internal neutrino lines using the formalism of Quantum Statistical Field Theory (more commonly and inappropriately called Finite Temperature Field Theory). For the purpose of determining the neutrino background contribution to $\Pi_{P}$ we take the background to consist only of neutrinos and antineutrinos, and therefore we will adopt the propagator corresponding to the vacuum for all the other particles. For a background characterized by a temperature $1 / \beta$ and chemical potential $\mu$, the propagator for massless neutrinos is given by

$$
\begin{aligned}
i S_{F}(p, \beta, \mu) & =\not p\left[\frac{i}{p^{2}}-2 \pi \delta\left(p^{2}\right) \eta_{F}(p, \beta, \mu)\right] \\
& =i S_{0}(p)+S^{\prime}(p, \beta, \mu),
\end{aligned}
$$

where $\eta_{F}$ contains the distribution functions for both neutrinos and antineutrinos:

$$
\eta_{F}(p, \beta, \mu)=\frac{\Theta(p \cdot u)}{e^{\beta(p \cdot u-\mu)}+1}+\frac{\Theta(-p \cdot u)}{e^{-\beta(p \cdot u-\mu)}+1} .
$$

Here, $\Theta$ denotes the step function which has the value unity for positive arguments, and is zero otherwise. Notice from Eq. (2.2) that the propagator has two parts. The first one, called $S_{0}$, is identical to the propagator in the vacuum. The second part, which has been denoted by $S^{\prime}$, is the correction due to the background neutrinos, and is non-zero only for on-shell neutrinos because of the factor $\delta\left(p^{2}\right)$.

Since the neutrinos do not couple to photons directly, the photon self-energy diagrams involving internal neutrino lines occur at the 2-loop level. These diagrams have been shown in Fig. 1. In drawing these, we have adopted the non-linear $R_{\xi}$ gauge [3] in which the $W w \gamma$ coupling vanishes, where $w$ is the unphysical Higgs which is eaten up by the $W$-boson in the unitary gauge.

Notice that each diagram contains only one internal neutrino line. Once we write down the amplitude corresponding to these diagrams, there will be two kinds of terms. The first kind, which will come from the vacuum part of the neutrino propagator, will give a contribution to the vacuum polarization. We are interested in the other contribution which depends on the background medium, involving the $S^{\prime}$ term of the neutrino propagator.

As we have already pointed out, the $S^{\prime}$-part of the propagator contributes only when the neutrino momentum $p$ satisfies the on-shell condition. If we cut the neutrino line so that it becomes a pair of external lines, then the resulting diagram contributes to the neutrino-photon forward scattering amplitude $M_{\mu \nu}(p, k)$. These diagrams are shown in Fig. 2. It then follows that, in the diagram where the neutrino line is closed, the background-dependent contribution 
to the photon self-energy, denoted by $\Pi_{\mu \nu}^{\prime}(k)$, is given by

$$
i \Pi_{\mu \nu}^{\prime}(k)=-\int \frac{d^{4} p}{(2 \pi)^{4}} \operatorname{Tr}\left[S^{\prime}(p) M_{\mu \nu}(p, k)\right],
$$

where the negative sign is due to the usual rule for a closed fermionic loop.

Therefore, our first task is to calculate $M_{\mu \nu}(p, k)$. For the moment, let us consider the diagrams for the non-forward scattering amplitude, and denote the momenta of the two external photons by $k$ and $k^{\prime}$. Since the external lines in those diagrams correspond to the neutrinos, the photon is attached to internal lines only. As a result, the contribution of these diagrams is well-defined (i.e., non-singular) in the limits $k \rightarrow 0, k^{\prime} \rightarrow 0$. This, combined with the gaugeinvariance conditions

$$
k^{\mu} M_{\mu \nu}=0, \quad k^{\prime \nu} M_{\mu \nu}=0,
$$

implies that the amplitude must be of order $k k^{\prime}$. In other words, the amplitude must be proportional to at least one power of $k$ and one power of $k^{\prime}$. Since $k=k^{\prime}$ for the forward scattering amplitude, we thus conclude that $M_{\mu \nu}(p, k)$ must be of order $k^{2}$.

This fact simplifies our task for the following reason. Except for the diagram in Fig. 2a, in all the other diagrams that contribute to the forward $\nu$ - $\gamma$ scattering the terms that have at least two powers of external momenta are of order $1 / M_{W}^{4}$ and higher, which are not important for small values of $k$ compared to $M_{W}$. Those diagrams also have terms of order $1 / M_{W}^{2}$, but they contain only one power of external photon momentum and, as argued above, by gauge invariance they will cancel out at the end. On the other hand the diagram in Fig. $2 \mathrm{a}$ can give a term that contains at least two powers of the photon momentum and at the same time is not necessarily of order $1 / M_{W}^{4}$. Denoting by $m$ the mass of the charged lepton in the loop, that term is of order $1 /\left(M_{W}^{2} m^{2}\right)$ for $\left|k^{2}\right|<m^{2}$. Thus, in order to determine the leading contribution (in powers of $M_{W}^{2}$ ) to the gauge invariant part of the amplitude we need to calculate only the diagram shown in Fig. 22a, retaining from that only the terms of order $1 / M_{W}^{2}$ that contain at least two powers of $k$. The result so obtained is proportional to $1 /\left(M_{W}^{2} m^{2}\right)$ and has significance in the kinematic regime $\left|k^{2}\right|<m^{2}$. For the kinematic regime $\left|k^{2}\right|>m^{2}$, of course, we need to calculate all the diagrams shown in Fig. 2.

\section{Details of the calculation}

For the purpose of calculation, it is again convenient to start with the general process

$$
\nu(p)+\gamma(k) \rightarrow \nu\left(p^{\prime}\right)+\gamma\left(k^{\prime}\right)
$$




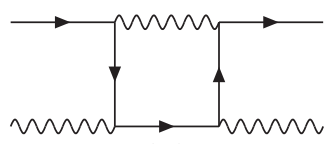

(a)

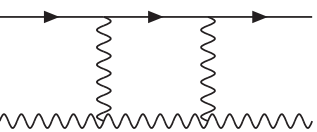

(e)

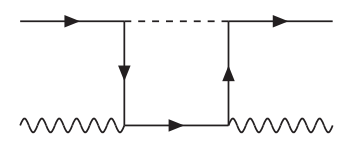

(b)

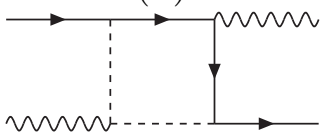

(d)

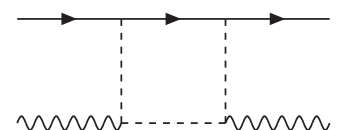

(f)

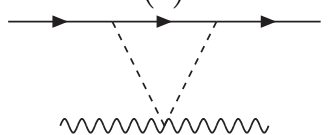

(h)

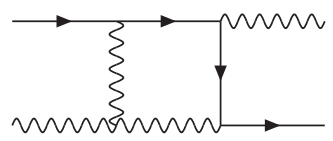

(c)

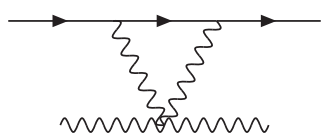

(g)

Figure 2: One-loop diagrams for neutrino-photon forward scattering, obtained by cutting the neutrino line in the diagrams of Fig. 11. In these diagrams, the external fermion lines are neutrinos. All other lines are as explained in the caption of Fig. 1. There are also crossed diagrams obtained by interchanging the external photon lines. 
and put $k=k^{\prime}$ at the end. The amplitude for this process coming from Fig. 2a can be written as

$$
M_{\mu \nu}^{(a)}=-\left(\frac{i g}{\sqrt{ } 2}\right)^{2}(-i e)^{2} \gamma_{\lambda} L\left\{T_{\lambda \mu \nu}\left(k, k^{\prime}\right)+T_{\lambda \nu \mu}\left(-k^{\prime},-k\right)\right\}
$$

where $L=\left(1-\gamma_{5}\right) / 2$, and

$$
\begin{aligned}
T_{\lambda \mu \nu}\left(k, k^{\prime}\right) & =\operatorname{Tr} \int \frac{d^{4} q}{(2 \pi)^{4}} L\left(\frac{i}{\not q+\not k-m}\right) \gamma_{\mu}\left(\frac{i}{\not q-m}\right) \gamma_{\nu}\left(\frac{i}{\not q+\not k^{\prime}-m}\right) \gamma_{\lambda} \\
& \times\left(\frac{-i}{\left(q-p+k^{\prime}\right)^{2}-M_{W}^{2}}\right),
\end{aligned}
$$

$m$ being the mass of the charged lepton in the loop. In writing this, we have adopted the Feynman gauge for the $W$ propagator, and we have used the relation

$$
\gamma^{\alpha} L A \gamma_{\alpha} L=-\gamma^{\alpha} L \operatorname{Tr}\left(A \gamma_{\alpha} L\right)
$$

which is valid for any $4 \times 4$ matrix $A$. Adopting a different gauge for the $W$ propagator, as well as including the diagram involving the exchange of the corresponding unphysical Higgs particle, would result in extra contributions of order $1 / M_{W}^{4}$ which, as already argued, we are not keeping. After some manipulations we obtain

$$
\begin{aligned}
& T_{\lambda \mu \nu}\left(k, k^{\prime}\right)+T_{\lambda \nu \mu}\left(-k^{\prime},-k\right)= \\
& \quad \operatorname{Tr} \int \frac{d^{4} q}{(2 \pi)^{4}}\left(\frac{1}{\not q+\not k-m}\right) \gamma_{\mu}\left(\frac{1}{\not q-m}\right) \gamma_{\nu}\left(\frac{1}{\not q+\not k^{\prime}-m}\right) \gamma_{\lambda} \\
& \quad \times \quad\left(\frac{R}{(q+p+k)^{2}-M_{W}^{2}}-\frac{L}{\left(q-p+k^{\prime}\right)^{2}-M_{W}^{2}}\right) .
\end{aligned}
$$

At this stage, we can put $k=k^{\prime}$, and replace the denominators appearing in the $W$ propagators by $q^{2}-M_{W}^{2}$, because the extra terms will contribute only to order $1 / M_{W}^{4}$. The rest of the calculation is straightforward and, using the convention

$$
\varepsilon_{0123}=-1,
$$

we obtain

$$
M_{\mu \nu}=\frac{-i e^{2} g^{2}}{8 \pi^{2} M_{W}^{2} m^{2}} \gamma_{\lambda} L\left(i k^{2} \varepsilon_{\mu \nu \lambda \rho} k^{\rho}\right) J\left(k^{2}\right)
$$

where

$$
J\left(k^{2}\right)=\int_{0}^{1} d x \frac{x(1-x)}{1-x(1-x)\left(k^{2} / m^{2}\right)} .
$$


Substituting Eq. (3.7) in Eq. (2.4) and using the background-dependent part of the neutrino propagator from Eq. (2.2) this gives

$$
i \Pi_{\mu \nu}^{\prime}(k)=\frac{-i e^{2} g^{2}}{8 \pi^{2} M_{W}^{2} m^{2}}\left(i k^{2} \varepsilon_{\mu \nu \lambda \rho} k^{\rho}\right) J\left(k^{2}\right) I_{\lambda},
$$

where

$$
I_{\lambda}=\int \frac{d^{4} p}{(2 \pi)^{4}} \operatorname{Tr}\left(\not p \gamma_{\lambda} L\right) 2 \pi \delta\left(p^{2}\right)\left[\frac{\Theta(p \cdot u)}{e^{\beta(p \cdot u-\mu)}+1}+\frac{\Theta(-p \cdot u)}{e^{-\beta(p \cdot u-\mu)}+1}\right] .
$$

Carrying out the trace, and performing the integration over $p_{0}$ in the rest frame of the medium with the help of the $\delta$-function, we obtain

$$
I_{\lambda}=\left(n_{\nu}-n_{\bar{\nu}}\right) u_{\lambda},
$$

where $n_{\nu, \bar{\nu}}$ stand for the number densities of neutrinos and antineutrinos in the rest frame of the medium. They are given by

$$
n_{\nu, \bar{\nu}}=\int \frac{d^{3} p}{(2 \pi)^{3}} \frac{1}{e^{\beta(|\vec{p}| \mp \mu)}+1},
$$

with the upper (lower) sign holding for neutrinos (antineutrinos), respectively.

This result can of course be used in any frame as long as we keep in mind that the number densities of the neutrinos and antineutrinos refer to the rest frame of the medium. Putting this back in Eq. (3.9) and comparing with the definition of $\Pi_{P}$ from Eqs. (1.2) and (1.5), we finally obtain

$$
\Pi_{P}^{(\nu)}(k)=\frac{e^{2} g^{2} k^{2} K}{8 \pi^{2} M_{W}^{2} m^{2}}\left(n_{\nu}-n_{\bar{\nu}}\right) J\left(k^{2}\right),
$$

where $K$ was defined in Eq. (1.6). We have added a superscript $\nu$ to $\Pi_{P}$ in Eq. (3.13) to remind us of the fact that this is the contribution to $\Pi_{P}$ from one neutrino specie in the background (see below).

As for $J\left(k^{2}\right)$, we can neglect the $k^{2}$-dependent term in the denominator if we are dealing with small $k^{2}$. The integration then gives

$$
J\left(k^{2}\right)=\frac{1}{6} .
$$

and Eq. (3.13) reduces to

$$
\Pi_{P}^{(\nu)}=\frac{\sqrt{2} G_{F} \alpha}{3 \pi}\left(\frac{k^{2}}{m^{2}}\right)\left(n_{\nu}-n_{\bar{\nu}}\right) K
$$

So from far we have assumed that the background contains neutrinos of only one flavor, which we have denoted generically by $\nu$. In general the background 
contains neutrinos of various flavors, and each one gives a contribution to $\Pi_{P}$ which is of the form given in Eq. (3.15). Notice however that this result for $\Pi_{P}^{(\nu)}$ depends on the mass $m$ of the charged fermion in the loop. Since the neutrinos do not have any mass or mixing in the standard model, the internal charged lepton is the electron if the thermal neutrino line corresponds to $\nu_{e}$, or either the muon or the tau if the thermal neutrino line corresponds to $\nu_{\mu}$ or $\nu_{\tau}$, respectively. We thus see that the contribution to $\Pi_{P}$ coming from the asymmetries of $\nu_{\mu}$ and $\nu_{\tau}$ are negligible since they are inversely proportional to the corresponding charged lepton mass. Therefore, for all practical purposes

$$
\Pi_{P}=\frac{\sqrt{2} G_{F} \alpha}{3 \pi}\left(\frac{k^{2}}{m_{e}^{2}}\right)\left(n_{\nu_{e}}-n_{\bar{\nu}_{e}}\right) K
$$

As already argued, the formula derived above is useful in the kinematic regime $\left|k^{2}\right|<m^{2}$ because it gives the leading contribution to $\Pi_{P}$, of order $1 / M_{W}^{2}$, in that regime. On the other hand, it is not valid for $\left|k^{2}\right|>m^{2}$ because terms of the same order $\left(k / M_{W}^{2}\right)$, which eventually all cancel, have been omitted. Therefore, a valid calculation for $\left|k^{2}\right|>m^{2}$ must include all the diagrams shown in Fig. 2. We have in fact performed that, and the result of that task is presented in the rest of this section for the sake of completeness. The reader interested in the physical implications of our results may skip this part and go to the next section.

For the purpose of compactness of notation, let us denote the amplitude of the diagram $A$ in the form

$$
\Pi_{\mu \nu}^{\prime}{ }^{(A)}=3 g^{2} e^{2}\left(n_{\nu}-n_{\bar{\nu}}\right) i \varepsilon_{\mu \nu \lambda \rho} u^{\lambda} k^{\rho} \int_{0}^{1} d x \int_{0}^{1-x} d y \int \frac{d^{4} q}{(2 \pi)^{4}} F^{(A)} .
$$

The task is now to present the quantities $F^{(A)}$ for each diagram. We set the gauge parameter $\xi$ of the non-linear $R_{\xi}$ gauge to be equal to unity. The complete Feynman rules for this choice can be obtained in Ref. [4]. Using these, it is easy to see that diagrams $2 \mathrm{f}-\mathrm{h}$ give no contribution at all. For the other ones, we obtain

$$
\begin{aligned}
& F^{(a)}=\frac{x\left\{2(1-3 y) q^{2}+4(1+y) m^{2}+4 y^{2}(1-y) k^{2}\right\}}{\left(q^{2}-A^{2}\right)^{4}} \\
& F^{(b)}=-\frac{r x\left\{(1-3 y) q^{2}+2(1+y) m^{2}+2 y^{2}(1-y) k^{2}\right\}}{\left(q^{2}-A^{2}\right)^{4}} \\
& F^{(c)}=\int_{0}^{1-x-y} d z \frac{\left\{-2 q^{2}+8 m^{2}+8(x+z)(1-x-z) k^{2}\right\}}{\left(q^{2}-B^{2}\right)^{4}}, \\
& F^{(d)}=-\frac{r q^{2}}{\left(q^{2}-B^{2}\right)^{4}}, \\
& F^{(e)}=\frac{8 x\left(q^{2}+y k^{2}\right)}{\left(q^{2}-C^{2}\right)^{4}}
\end{aligned}
$$


where

$$
r=\left(m / M_{W}\right)^{2}
$$

and

$$
\begin{aligned}
& A^{2}=(1-x-y) M_{W}^{2}+(x+y) m^{2}-y(1-y) k^{2} \\
& B^{2}=(1-x-y) M_{W}^{2}+(x+y) m^{2}-(x+z)(1-x-z) k^{2} \\
& C^{2}=(x+y) M_{W}^{2}+(1-x-y) m^{2}-y(1-y) k^{2}
\end{aligned}
$$

It is straight forward (though tedious) to show that if $k^{2}=0$, the sum of all the diagrams vanish. This is in accordance with the argument given earlier, showing that the forward scattering amplitude must be at least of order $k^{2}$.

For small $k^{2}$, the integrals can be expanded in power series of $k^{2}$, and the integrations of at least the $O\left(k^{2}\right)$-term can be performed analytically. This gives, in the notation introduced in Eq. (3.7),

$$
\begin{aligned}
J\left(k^{2}\right)= & \frac{1}{24(1-r)^{3}}\left[4-20 r+19 r^{2}-9 r^{3}-\frac{r \ln r}{1-r}\left(8-12 r+12 r^{2}-4 r^{3}\right)\right] \\
& +O\left(k^{2}\right),
\end{aligned}
$$

For small $r$, it is easy to see that this reduces to the value given in Eq. (3.14).

\section{Dispersion relations and optical activity}

The bilinear terms in the effective Lagrangian, which describes the propagation of electromagnetic waves through a medium, may be written in momentum space in the form

$$
\mathcal{L}=-\frac{1}{4} F_{\mu \nu}^{*} F^{\mu \nu}+\mathcal{L}^{\prime}
$$

The first term in Eq. (4.1) is the standard kinetic energy term for the electromagnetic field, while $\mathcal{L}^{\prime}$ is the contribution from the background medium, which is given in terms of the photon self-energy by

$$
\mathcal{L}^{\prime}=\frac{1}{2} A^{* \mu} \Pi_{\mu \nu} A^{\nu}
$$

In physical applications we must include the effect of the charged plasma along with that of the neutrinos. While the main contribution to $\Pi_{P}$ is due to the neutrino background, as determined above, $\Pi_{T, L}$ receive their main contribution from the electron background. For our purposes, it is sufficient to recall the well known result

$$
\Pi_{T, L}(\omega, K \rightarrow 0)=\omega_{P}^{2}
$$


where $\omega_{P}^{2}$ is the plasma frequency. Eq. (4.3) holds in the limit $K, \omega \ll m_{e}$ and it is useful for finding the dispersion relations of the propagating modes in the long wavelength limit. In general,

$$
\omega_{P}^{2}=4 e^{2} \int \frac{d^{3} p}{(2 \pi)^{3} 2 E}\left(f_{e}+f_{\bar{e}}\right)\left[1-\frac{p^{2}}{3 E^{2}}\right],
$$

where $f_{e, \bar{e}}$ are the electron and positron distribution functions. When the electron gas is in the non-relativistic limit, Eq. (4.4) reduces to the standard formula

$$
\omega_{P}^{2}=\frac{e^{2} n_{e}}{m_{e}},
$$

where $n_{e}$ is the electron number density. The equation of motion that follows from Eqs. (4.1) and (4.2) is

$$
D_{\mu \nu}^{-1} A^{\nu}=0
$$

where

$$
D_{\mu \nu}^{-1}=\left(-k^{2}+\Pi_{T}\right) R_{\mu \nu}+\left(-k^{2}+\Pi_{L}\right) Q_{\mu \nu}+\Pi_{P} P_{\mu \nu} .
$$

This equation may be written in terms of the components of $A^{\mu}$ by means of the following device. We introduce two vectors whose components in the rest frame of the medium are $\left(0, \hat{e}_{1,2}\right)$, and where $\hat{e}_{1,2}$ are unit vectors orthogonal to $\vec{k}$ with the convention that $\hat{e}_{1}, \hat{e}_{2}, \hat{k}$ form a right-handed coordinate system. Then, as shown in Ref. [2], in any frame these vectors satisfy

$$
e_{1 \mu}=i P_{\mu \nu} e_{2}^{\nu}, \quad e_{2 \mu}=-i P_{\mu \nu} e_{1}^{\nu} .
$$

It is also useful to define

$$
e_{3}^{\mu}=\frac{\tilde{u}^{\mu}}{\sqrt{-\tilde{u}^{2}}},
$$

which is orthogonal to $e_{1,2}^{\mu}$ and $k^{\mu}$. If we now decompose $A^{\mu}$ in the form

$$
A^{\mu}=A_{1} e_{1}^{\mu}+A_{2} e_{2}^{\mu}+A_{L} e_{3}^{\mu}+A_{\|} k^{\mu}
$$

and substitute this in Eq. (4.6), it follows that the component $A_{\|}$decouples from the equations, as it should be since it is associated with the gauge degree of freedom, while for the longitudinal component we obtain the equation

$$
\left(-k^{2}+\Pi_{L}\right) A_{L}=0
$$

which implies the dispersion relation

$$
k^{2}=\Pi_{L} .
$$


On the other hand, for the transverse modes we obtain a set of coupled equations that can be written in matrix form as

$$
\left(\begin{array}{cc}
-k^{2}+\Pi_{T} & -i \Pi_{P} \\
i \Pi_{P} & -k^{2}+\Pi_{T}
\end{array}\right)\left(\begin{array}{c}
A_{1} \\
A_{2}
\end{array}\right)=0 .
$$

In this way, the dispersion relation for the transverse modes is found to be given by the solutions of the equations

$$
k^{2}=\Pi_{T} \pm \Pi_{P}=\omega_{P}^{2} \pm \Pi_{P}
$$

with the corresponding polarization vectors

$$
e_{\mu}^{( \pm)}=\frac{1}{\sqrt{2}}\left(e_{1 \mu} \pm i e_{2 \mu}\right) .
$$

Thus, the general solution for $A^{\mu}$ is of the form

$$
A_{\mu}=A_{+} e_{\mu}^{(+)}+A_{-} e_{\mu}^{(-)}+A_{L} e_{3 \mu},
$$

with $A_{ \pm}$representing the amplitudes of the left(-) and right(+) circularly polarized modes.

In this way we see that the two transverse modes have different dispersion relations. This is just the phenomenon of optical activity, which in the present case is induced by the chiral nature of the neutrino interactions. The dispersion relation for each mode can be obtained explicitly by solving Eq. (4.14). For this purpose, it is useful rewrite the expression for $\Pi_{P}$ as

$$
\Pi_{P}=a R_{\nu} k^{2} K,
$$

where

$$
R_{\nu} \equiv \frac{n_{\nu_{e}}-n_{\bar{\nu}_{e}}}{n_{0}}
$$

is the dimensionless form of the neutrino asymmetry, and

$$
a \equiv \frac{\sqrt{2} G_{F} \alpha}{3 \pi}\left(\frac{n_{0}}{m_{e}^{2}}\right)
$$

for any arbitrary benchmark value $n_{0}$. Taking $n_{0}=1 \mathrm{~cm}^{-3}$, we obtain

$$
a=4 \times 10^{-43} \mathrm{GeV}^{-1}=8 \times 10^{-57} \mathrm{~cm} .
$$

The solutions to Eq. (4.14) can be obtained to the leading order in $a$ (i.e, leading order in $G_{F}$ ) by substituting $k^{2} \simeq \omega_{P}^{2}$ on the right hand side of Eq. (4.17). In this way we find the dispersion relations of the two modes to be given by

$$
\omega_{ \pm}(K)=\sqrt{K^{2}+\omega_{P}^{2}} \pm \frac{1}{2}\left(a R_{\nu} \omega_{P}^{2}\right) \frac{K}{\sqrt{K^{2}+\omega_{P}^{2}}} .
$$


An alternative interpretation of these dispersion relations is that, for a given value of $\omega$, the two circularly polarized modes travel with different momenta

$$
K_{ \pm}=\left(\omega^{2}-\omega_{P}^{2}\right)^{1 / 2} \mp \frac{1}{2} a R_{\nu} \omega_{P}^{2} .
$$

In any case, it follows that the two modes have different phase and group velocities. This fact can manifest itself in various ways, some of which we discuss in the next section.

\section{Applications}

\subsection{Differential time delay}

Consider a pulse of light originating at some particular instant and subsequently propagating through the neutrino medium. Since the two circularly polarized components have different group velocities $v_{g}^{ \pm}=\partial \omega_{ \pm} / \partial K$, they will disperse in time into two separate pulses. If the time delay between them is larger than the width of the original pulse, then it can be experimentally observable.

From the dispersion relations in Eq. (4.21) we obtain

$$
\begin{aligned}
v_{g}^{ \pm} & =\frac{K}{\sqrt{K^{2}+\omega_{P}^{2}}} \pm \frac{1}{2} a R_{\nu} \frac{\omega_{P}^{4}}{\left(K^{2}+\omega_{P}^{2}\right)^{3 / 2}} \\
& \simeq 1-\frac{\omega_{P}^{2}}{K^{2}} \pm \frac{a R_{\nu} \omega_{P}^{4}}{2 K^{3}},
\end{aligned}
$$

where, in the second formula, we have indicated the approximate result in the limit $K \gg \omega_{P}$. At a distance $\ell$ from the emission point, the differential time delay between the two pulses is given by

$$
\begin{aligned}
\Delta t & =\left|\frac{\ell}{v_{g}^{+}}-\frac{\ell}{v_{g}^{-}}\right| \\
& \simeq \frac{a R_{\nu} \omega_{P}^{4} \ell}{K^{3}},
\end{aligned}
$$

where in the final step we have used the approximate formula for $v_{g}^{ \pm}$given in Eq. (5.1).

As an example of a realistic setting where these considerations may be relevant, we mention the following. Gamma ray burster (GRB) photons have an average fluence $F=10^{-6} \mathrm{erg} / \mathrm{cm}^{2}$, per burst. If they are at cosmological distances [5] $D=3000 \mathrm{Mpc}$ then the total energy output per burst $E=4 \pi D^{2} F=10^{51}$ ergs. Since this is equal to the gravitational binding energy of a typical neutron star, they are thought to arise from the merger of neutron stars. The gravitational energy of the neutron stars gets converted into kinetic energy in the form of an expanding fireball. A temperature of the fireball of about $10 \mathrm{MeV}$ 
explains the observed spectrum of the signal. From the average time variability of the burst which is $1-10 \mathrm{~s}$, the size of the radiation zone is expected to be $10^{11}$ to $10^{12} \mathrm{~cm}$. Assuming that the neutrinos are produced by a shift in the beta equilibrium of the neutron star, we can have $n_{e} \sim n_{\nu} \sim 10^{3} \mathrm{MeV}^{3}$. The differential time delay between the right and the left polarized components of a burst signal of mean energy $1 \mathrm{MeV}$, traveling through this medium will have a differential time lag $\Delta t \sim 10^{-6} \mathrm{~s}$, which may lie within the observable range.

\subsection{Wavelength independent optical rotation}

As our second example, consider a monochromatic wave propagating along a given direction, which we take to be the $z$-axis. If the wave is linearly polarized (that is, it contains an equal admixture of the two circular polarizations), then the amplitude of such a wave is of the form

$$
\begin{aligned}
\vec{A}(z, t) & =A_{\omega} e^{-i \omega t}\left(e^{i z K_{+}} \hat{e}^{(+)}+e^{i z K_{-}} \hat{e}^{(-)}\right) \\
& =A_{\omega} e^{-i \omega t} e^{i \frac{1}{2}\left(K_{+}+K_{-}\right) z}\left(e^{-i \frac{1}{2}\left(K_{-}-K_{+}\right) z} \hat{e}^{(+)}+e^{i \frac{1}{2}\left(K_{-}-K_{+}\right) z} \hat{e}^{(-)}\right) .
\end{aligned}
$$

We have implicitly chosen the origin and orientation of the coordinate system in such a way that, at $z=0$, the linear polarization vector of the wave points along $\hat{e}_{1}$, or the $x$-direction. Using the relations $\hat{e}^{( \pm)}=\left(\hat{e}_{1} \pm i \hat{e}_{2}\right) / \sqrt{2}$ implied by Eq. (4.15), it follows that at any given distance $z=\ell$ the polarization vector of the wave points at an angle given by

$$
\phi(\ell)=\frac{1}{2}\left(K_{-}-K_{+}\right) \ell=\frac{1}{2} a R_{\nu} \omega_{P}^{2} \ell
$$

relative to the $x$-axis.

It is known that such a rotation of the polarization vector is also caused by the presence of an external magnetic field. This effect, known as the Faraday rotation, is commonly used for measuring extragalactic magnetic fields. The difference between the optical rotation caused by neutrinos and the Faraday rotation effect is that the expression for $\phi$ given in Eq. (5.4) is independent of the wavelength $\lambda$ of the propagating waves, whereas in the case of the Faraday rotation the angle is proportional to $\lambda^{2}$.

For comparison with other sources of optical activity, it is useful to note that the contribution from the neutrino background to the effective Lagrangian given in Eq. (4.2) can be written in the form

$$
\mathcal{L}_{P}=\frac{1}{2} A^{* \mu} S^{\nu} \tilde{F}_{\mu \nu},
$$

where $\tilde{F}^{\mu \nu} \equiv(1 / 2) \epsilon^{\mu \nu \alpha \beta} F_{\alpha \beta}$ is the dual electromagnetic tensor and

$$
S^{\mu}=\frac{\Pi_{P}}{K} u^{\mu}
$$




$$
=a R_{\nu} k^{2} u^{\mu} \simeq a R_{\nu} \omega_{P}^{2} u^{\mu} .
$$

In the second line of Eq. (5.6) we have used Eq. (4.17), and we have also put $k^{2} \simeq \omega_{P}^{2}$ which is correct to leading order in $G_{F}$. It has been pointed out in the literature that such an interaction breaks the invariance under the $C P T$ transformation [6], and also breaks Lorentz invariance [7, 8] if $S_{\mu}$ is a fixed 4-vector (i.e., $S_{\mu}$ does not transform as a four-vector under Lorentz transformations). As we have indicated above, one distinctive feature of this term is a rotation of the polarization of electromagnetic waves by an angle which is identical for all wavelengths. Using the observations about the polarization of the radiation from distant sources, various limits have been deduced on possible deviations from the physics of the standard model [4, 9, 10, 11]. In addition, it has been recently claimed 12] that the effect induced by a term of the kind given in Eq. (5.5) is actually observed in the data, indicating that the radiation from radio galaxies is polarized along a preferential axis with respect to the galaxy [13. This claim has been contested by several authors [14, and it has been argued that the data should instead be interpreted as an upper bound on the possible non-magnetic optical rotation.

We want to point out here that the effect of a wavelength-independent optical rotation occurs even in the standard model of particle interactions, if the universe has an unequal number of neutrinos and antineutrinos. Moreover, it is present even if the neutrinos are strictly massless. This was pointed out earlier in a qualitative manner in Ref. [2]. The new input of the present work is to specify that the magnitude of $S_{\mu}$ in such a case is given by Eq. (5.6). An estimate of it can be obtained as follows.

If the cosmological neutrino background has a non-zero chemical potential, then it is bounded by the constraint that the neutrino energy density should not exceed the closure density of the universe, $\mu_{\nu} / 8 \pi^{2}<2 \times 10^{-47} \mathrm{GeV}^{4}$, which means that $R_{\nu}=\mu_{\nu}^{3} /\left(6 \pi^{2} n_{0}\right) \leq 5 \times 10^{5}$. With $\omega_{P}^{2}=4 \pi \alpha n_{e} / m_{e}$, taking $n_{e}=$ $0.03 \mathrm{~cm}^{-3}=2.3 \times 10^{-43} \mathrm{GeV}^{3}$ in the intergalactic medium and $\ell=H_{0}^{-1}=$ $0.5 \times 10^{42} \mathrm{GeV}$ the magnitude of the optical rotation given by Eq. (5.4) is of order $10^{-36}$. This seems to be too small to be observable. Therefore, if the observation of a wavelength-independent optical rotation is firmly established, then it probably cannot be due to the cosmological background neutrinos, at least within the assumptions of the standard particle physics and cosmological models.

\subsection{Differential bending of light}

Consider the bending of light by a massive body in a neutrino medium. This could occur in the halo of a galaxy, in the exterior of a neutron star or the neutrino-sphere of a supernova. In empty space, a light ray bends by an angle $\theta=4 G M / b$, where $M$ is the mass of the body and $b$ is the impact parameter. On the other hand it has been shown that the gravitational bending angle for 
a massive spinless particles depends on the mass of the particle [15]. This suggests that the bending angle for a photon propagating in a matter background depends on the dispersion relation of the photon. In fact, it has been found that interactions of the type given in Eq. (5.5) indeed give polarization-dependent bending for light [10, 11].

Thus, when light propagates in a neutrino medium, the left and the right polarized components bend through different angles, which we denote by $\theta_{ \pm}$. The image of the source splits into two images, separated by an angle $\Delta \theta=$ $\theta_{+}-\theta_{-}$. To calculate the bending angle we use the method given in Ref. [16], adapting it to the present case in which $\omega \neq K$. The starting point is the equation of motion for a light wave of momentum $k^{\mu}$ which, in the limit of geometrical optics, is

$$
d k_{\mu}=\frac{1}{2}\left(\partial_{\mu} h^{\alpha}{ }_{\beta}\right) k_{\alpha} d x^{\beta},
$$

where

$$
h_{\alpha \beta}=g_{\alpha \beta}-\eta_{\alpha \beta}
$$

with $\eta_{\alpha \beta}$ being the flat-space metric. As in Ref. [16], we consider a ray of light with a given polarization $\lambda= \pm$, passing by a gravitational body of mass $M$ with impact parameter $b$. Taking the $z$-axis along the direction of incidence, we then have

$$
\begin{aligned}
d x^{\beta} & =\left(\frac{1}{v_{\lambda}}, 0,0,1\right) d z \\
k^{\alpha} & =\left(1,0,0, v_{\lambda}\right) \omega,
\end{aligned}
$$

where $v_{\lambda}=K_{\lambda}(\omega) / \omega$. The bending angle is given by

$$
\theta_{\lambda}=\frac{\Delta k_{x}}{k_{z}}
$$

where $\Delta k_{x}$ is the total change in $k_{x}$. Using the expression for $h_{\alpha \beta}$ corresponding to the Schwarschild metric and using Eqs. (5.7) and (5.9), we obtain

$$
\Delta k_{x}=\frac{G M b \omega}{v_{\lambda}}\left(1+v_{\lambda}^{2}\right) \int_{-\infty}^{\infty} d z \frac{1}{\left(z^{2}+b^{2}\right)^{3 / 2}}=\frac{2 G M \omega}{b v_{\lambda}}\left(1+v_{\lambda}^{2}\right),
$$

and from Eq. (5.10

$$
\theta_{\lambda}=\frac{2 G M}{b}\left(1+\frac{1}{v_{\lambda}^{2}}\right) .
$$

Therefore, the bending angles for the two polarizations differ by an amount

$$
\Delta \theta \equiv \theta_{+}-\theta_{-}=\frac{2 G M}{b}\left(\frac{1}{v_{+}^{2}}-\frac{1}{v_{-}^{2}}\right) .
$$


Remembering that $v_{ \pm}=K_{ \pm}(\omega) \omega$ and using Eq. (4.22), we then find that

$$
\Delta \theta \simeq \frac{4 G M}{b} \frac{a R_{\nu} \omega_{P}^{2} \omega^{2}}{\left(\omega^{2}-\omega_{P}^{2}\right)^{3 / 2}}
$$

for the neutrino background.

\section{Conclusions}

A background in which the number of neutrinos and antineutrinos are not the same is an optically active medium. This observation, first pointed out in Ref. [2], has several implications and could in principle lead to potentially important applications. In this paper we have presented explicitly the results of the calculation of the neutrino background contribution to the photon self-energy in such a medium. This has allowed us to deduce the photon dispersion relations for the two circularly polarized states of the photon traversing the neutrino background, and analyze in some detail several of the possible physical effects. We have shown, in particular, that the neutrino background induces a difference in the time delay between the light signals of opposite polarizations, which could lie in an observable range for photons originating in gamma ray bursters. We have also shown that the optical rotation effect caused by the neutrinos is independent of the wavelength of the electromagnetic wave, in contrast with the Faraday effect caused by the presence of a magnetic field, which depends on the square of the wavelength. In addition, we derived the formula for the bending angle of a light ray passing by a massive object embedded in the neutrino background, taking into account that the photon dispersion relation is not the same as in the vacuum, and that it is in fact different for each polarization mode.

At first sight, all these effects tend to be small. However, we point out once more that the calculations that we have described, and the results that we have obtained, do not depend on any physical assumptions beyond those required by the standard model of particle interactions, including the question of whether or not the neutrinos have a non-zero mass. Hence, the effects that we have considered are present at some level. It would not be the first time that a seemingly unimportant feature of the electromagnetic interactions of neutrinos in a background medium, such as the neutrino index of refraction of a neutrino in the presence of a magnetic field [17], finds its way much later to become the focus of an important problem [18]. In the presents case, we have considered several consequences related to the optically active (chiral) nature of a neutrino background, some of which could be observationally testable, and should in any case be kept in mind.

This work have been partially supported by the US National Science Foundation Grant PHY-9600924 (J.F.N). 


\section{References}

[1] H. A. Weldon, Phys. Rev. D26, 1394 (1982).

[2] J.F. Nieves and P.B. Pal, Phys. Rev. D39, 652 (1989); ibid.40, 2148E (1989).

[3] M. B. Gavela et al, Nucl. Phys. B257, 1993 (1981).

[4] J. F. Nieves, P. B. Pal and D. G. Unger, Phys. Rev. D28, 908 (1983).

[5] B.Paczynski, Ap. J. Lett. 317, L51 (1987).

[6] J.F. Nieves and P.B. Pal, Phys. Rev. D40, 1350 (1989).

[7] S.M.Carroll, G.B.Field and R.Jackiw, Phys. Rev. D41, 1231 (1990).

[8] S. Coleman and S. L. Glashow, Phys. Lett. B405, 249 (1997).

[9] D. Harari and P. Sikivie, Phys. Lett. B289, 67 (1992).

[10] S.Mohanty and S.N.Nayak, Phys. Rev. D48, 1526 (1993).

[11] S.Mohanty and S.N.Nayak, Phys. Rev. Lett. 70, 4038 (1993).

[12] B. Nodland and J. P. Ralston, Phys. Rev. Lett. 78, 3043 (1997).

[13] D.J.Saika and C.J.Salter, Annu. Rev. Astron. Astrophys. 26, 93 (1986). J.N.Clarke et al, MNRAS 190, 205 (1980); P.Haves and R.G.Conway, MNRAS 173, 53 (1975).

[14] J.F.C. Wardle, R.A. Perley and M.H. Cohen, Phys. Rev. Lett. 79, 1801 (1997); D. J. Eisenstein and E. F. Bunn, Phys. Rev. Lett. 79, 1957 (1997); S.M.Carroll and G.B.Field, Phys. Rev. Lett. 79, 2394 (1997);

[15] E. Golowich, P.S. Gribosky and P.B. Pal, Am. J. Phys. 58, 688 (1990).

[16] H. C. Ohanian, Gravitation and spacetime, (W. W. Norton and Company, New York 1976), page 120.

[17] J. C. D’Olivo, José F. Nieves and P. B. Pal, Phys. Rev. D40, 3679 (1989)

[18] A. Kusenko and G. Segrè, Phys. Rev. Lett. 77, 4872 (1996). 